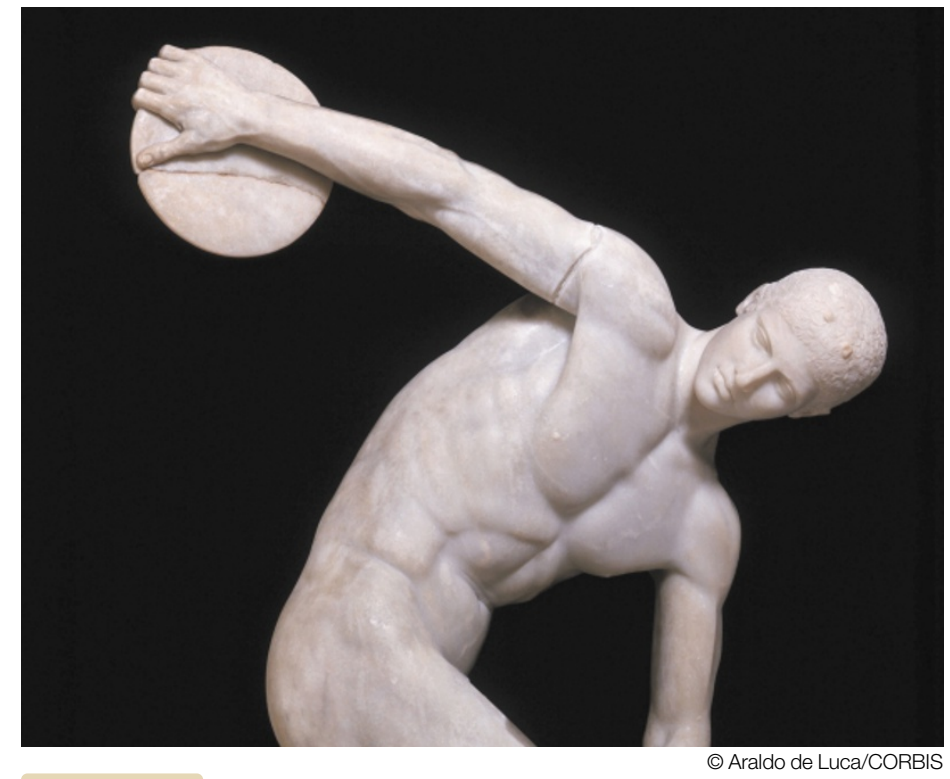

APOPTOSIS

\section{Death shapes life}

The developing mouse embryo is an exquisite molecular sculpture. Early on, digits and organs are shaped by a programmed cell death that bears the characteristic hallmarks of apoptosis. Paradoxically, however, organ sculpting is normal (albeit delayed) in embryos missing the usual apoptotic suspects such as caspase-9 and Apaf1. One explanation is that the embryo is shaped by a second, caspase-independent pathway, and a report in Nature now supports this idea.

A little over two years ago, Guido Kroemer, Josef Penninger and collaborators cloned and characterized apoptosis-inducing factor (AIF), a mitochondrial factor released in response to death-triggering stimuli. They now implicate AIF in developmental cell death by showing that genetic inactivation of AIF abolishes death in a model of early embryogenesis.

To do this, the authors first used homologous recombination to disrupt the aif gene in mouse embryonic stem (ES) cells. Then, to mimic the conditions of early development, they cultured aggregates of the AIF-deficient ES cells in the absence of leukaemia inhibitory factor and feeder cells. Under these conditions the ES cells develop into embryoid bodies multicellular aggregates containing an outer layer of endodermal cells and a solid core of undifferentiated ectodermal cells. Cells within this inner core then normally undergo a wave of programmed death in a process known as cavitation.

How does the lack of AIF affect cavitation? The authors found that, in embryoid bodies derived from the AIF-deficient ES cells, cavitation was blocked. Then, by checking for the incorporation of 5-bromodeoxyuridine, they confirmed that this apparent block to cell death was not due simply to increased cell proliferation.

During cavitation in wild-type embryoid bodies, caspase- 3 - a downstream effector of caspase9/Apaf-1 - is usually activated. To check the involvement of caspases in cavitation, Kroemer, Penninger and co-workers studied embryoid bodies derived from caspase- $-^{-1-}$ and apaf$1^{-1-}$ ES cells. In both cases, although caspase- 3 was no longer activated, cavitation proceeded as normal. The inference, then, is that the AIF-dependent cell death seen in cavitation is independent of caspase-dependent death pathways.

The idea of a second pathway is a controversial one. But the authors tentatively speculate that AIF may "represent a pathway of apoptosis that predates the caspase pathway". In support of this idea, they say, is the fact that AIF homologues have been identified in all metazoan phyla, whereas there is, as yet, no evidence for caspases in plants or in unicellular organisms that can nonetheless undergo programmed cell death.

Alison Mitchell

(2) References and links ORIGINAL RESEARCH PAPER Joza, N. et al. Essential role of the mitochondrial apoptosisinducing factor in programmed cell death. Nature 410, 549-554 (2001)

FURTHER READING Daugas, E. et al. Apoptosis-inducing factor (AIF): a ubiquitous mitochondrial oxidoreductase involved in apoptosis. FEBS Lett. 476, 118-123 (2000)
CELL SIGNALLING

\section{Two-way attraction}

Many ligands for cell-surface receptors are themselves transmembrane molecules. One idea is that this allows bidirectional signalling, a possibility that has been shown biochemically for B ephrins. Reporting in Cell, Lu, Sun and colleagues now elucidate a molecular basis for this reverse signalling through the identification of PDZ-RGS3 - a novel factor that couples B ephrins to a G-protein-coupled receptorand reveal a new mechanism for regulating cell chemoattraction.

To identify factors that might mediate reverse signalling for $B$ ephrins, the authors did a two-hybrid screen using the ephrin B2 cytoplasmic tail. From this, they pulled out a novel protein that contained a PDZ domain and an RGS domain. RGS proteins act as GTPase-activating proteins for heterotrimeric $\mathrm{G}$ proteins. This protein showed homology to human RGS3, so they called it PDZRGS3. Next, to confirm that this interaction was physiologically relevant, they showed that it occurred in an in vitro GST-fusion pulldown assay, and in co-immunoprecipitations from transfected cell lines and from endogenously expressed proteins in neural cell lines and the developing brain. Consistent with this, Lu, Sun and colleagues found, using in situ hybridization, that PDZ-RGS3 and $B$ ephrins are expressed together in mouse embryos.

What is the relationship between B ephrins and PDZ-RGS3? To address this, they used a Xenopus assay that had previously revealed a role for ephrin B1 in adhesion. Here, ephrin-B1's cytoplasmic tail causes de-adhesion. The authors found that PDZRG3 mediates the downstream effects of ephrin B1's cytoplasmic tail, and that both the PDZ and RGS motifs are important for this.

Given the presence of the RGS motif, Lu, Sun and colleagues predicted that downstream signalling from $B$ ephrins might regulate a G-protein-coupled receptor that mediates chemoattraction. To test this, they assessed the effects of soluble EphB2 receptor on chemoattractants for cerebellar granule cells. And they found that it specifically inhibited the effects of SDF-1 - a chemokine that signals through the G-proteincoupled receptor CXCR4.

The authors propose a model whereby PDZ-RGS3 provides the crucial link between these two signalling pathways, and allows ephrin B reverse signalling to antagonize the effects of SDF-1 on cells. Given the prevalence of PDZ domains in signalling proteins, they speculate that PDZ-RGS proteins might provide a pathway that controls widespread processes regulated by $\mathrm{G}$ proteins.

Alison Schuld

6) References and links ORIGINAL RESEARCH PAPER $L u$, Q. et al. Ephrin-B reverse signaling is mediated by a nove PDZ-RGS protein and selectively inhibits G-proteincoupled chemoattraction. Cell 105, 69-79 (2001) 